\title{
Superconductivity near Pomeranchuk instabilities in the spin channel
}

\author{
Daniel G. Barci \\ Departamento de Física Teórica, Universidade do Estado do Rio de Janeiro, Rua São Francisco \\ Xavier 524, 20550-013, Rio de Janeiro, RJ, Brazil. \\ Paulo S. A. Bonfim \\ Universidade UnigranRio, Rua Prof. José de Souza Herdy, 1.160, 25071-202, Duque de \\ Caxias, RJ, Brazil
}

\begin{abstract}
We study the competition between a Pomeranchuk instability in the spin channel with angular momentum $\ell=1$ and an attractive interaction, favoring Cooper-pair formation. We found that the superconducting gap strongly suppresses the phase space for the Pomeranchuk instability. We computed a mean-field phase diagram displaying a first order transition between two superconductor phases with different symmetries: p-wave (with spontaneously generated spin-orbit interaction) and s-wave for greater values of the coupling constant. Moreover, we have looked for a possible modulated superconducting phase. We have found that this phase appears only as a meta-stable state in the strong coupling regime.
\end{abstract}

Keywords: Fermi liquid instabilities; Pomeranchuk; Superconductivity

\section{Introduction}

A Fermi liquid is, except in one dimension, a very stable state of matter 1 . At least two types of instabilities, related with attractive interactions, are known: Pomeranchuk ${ }^{2}$ and superconducting instabilities. Pomeranchuk instabilities occur in the presence of two-body interactions containing a strong attractive component in the forward scattering channel with definite angular momentum. In the context of Landau theory, the instability sets in when one or more dimensionless Landau parameters $F_{\ell}^{s, a}$, with angular momentum $\ell$ in the charge $(s)$ or spin $(a)$ channel, acquire large negative values.

Pomeranchuk instabilities in a charge sector spontaneously break rotational symmetry ${ }^{3}$. In particular, an instability in the $F_{2}^{s}$ channel produces an ellipsoidal deformation of the Fermi surface $\frac{4,5}{5}$. From a dynamical point of view, the resulting anisotropic ground state is a non-Fermi liquid, due to over-damped Goldstone modes that wipe out quasi-particle excitations ${ }^{6.7 .8}$.

Instabilities in the spin channel are also very interesting. For instance, the ferromagnetic Stoner instability $\underline{\underline{9}}$ occurs when $F_{0}^{a}$ acquires large negative values, producing a divergence in the magnetic susceptibility. This phase transition pre- 
serves rotational symmetry, however it breaks time-reversal symmetry. On the other hand, it is possible to have spin instabilities that preserve time-reversal invariance 10,11. Higher order angular momentum interactions produce anisotropic as well as isotropic phases. Several examples were studied in detail ${ }^{12,13}$. The $\ell=1$ channel have special interest. When $F_{1}^{a}<0$, an ordered isotropic and time reversal invariant phase is possible. This phase, called $\beta$-phase in ref. [12], dynamically generates a spin-orbit coupling. This is a very interesting possibility, since it allows the generation of spin-orbit couplings from many-body correlations, differently from the usual one-particle relativistic effect.

On the other hand, superconductivity in the presence of spin-orbit interactions is nowadays of great interest 14 . Superconductivity is developed in the presence of a small attractive interaction in the particle-particle (BCS) channel. The superconducting (SC) state is generally characterized by a complex order parameter which breaks gauge symmetry, $\Delta_{\sigma, \sigma^{\prime}}\left(\vec{r}, \vec{r}^{\prime}\right)=\left\langle\psi_{\sigma}^{\dagger}(\vec{r}) \psi_{\sigma^{\prime}}^{\dagger}\left(\vec{r}^{\prime}\right)\right\rangle$, where the operator $\psi_{\sigma}^{\dagger}(\vec{r})$ creates an electron with spin $\sigma$ at the position $\vec{r}$. The usual classification of $\Delta_{\sigma, \sigma^{\prime}}\left(\vec{r}, \vec{r}^{\prime}\right)$ as s-wave, d-wave, p-wave, etc, resides in the irreducible representations of the lattice point group. Also, the absence of spin-orbit interactions allows the additional differentiation between singlet and triplet order parameters. However, the SC state could also break lattice translation and/or rotational symmetry. In that case, this classification is no more possible. One particular example is an oscillating order parameter like $\Delta_{\sigma, \sigma^{\prime}}(\vec{r}, 0)=\Delta_{\sigma, \sigma^{\prime}} \cos (\vec{q} \cdot \vec{r})$, where $\vec{q}$ is an ordering wavevector. In a modulated superconducting state, proposed by Fulde, Ferrell, Larkin and Ovchinnikov $\underline{15}$,16 (FFLO state), the spatial modulation of the Cooper pairing is due to a mismatch of Fermi surfaces, produced by an external magnetic field (by Zeeman effect). FFLO states have also been proposed to occur in other scenarios like in imbalanced cold atoms with different species 17 and in heavy fermions systems when different orbitals hybridize under external pressure $\frac{18}{}$. Also, for generally non-local BCS potentials, a modulated SC order parameter can coexist with charge density waves $\frac{19}{}$. Recently, a striped order parameter, called "Pair Density Wave, (PDW)", was proposed $20,21,22,23$ to explain anomalous transport properties observed in cuprates superconductors.

Although Pomeranchuk and BCS instabilities are generated by attractive interactions, they are competing ones. A superconducting gap suppresses Fermi surface deformations. A detailed example of this effect can be observed by considering the competition between d-wave Pomeranchuk instabilities and a d-wave superconducting order parameter 24,25 .

In the spin channel, due to time-reversal symmetry breaking, a stronger competition is expected. However, it is possible to have instabilities with higher angular momentum that preserve time-reversal invariance. In this paper, we analyze an example of this class of systems. In particular, the $\beta$-phase $\frac{12}{2}$ opens the possibility of the formation of Cooper pairs with zero helicity and finite momentum, possibly producing a modulated superconducting state.

The main point of this paper is to report on the competing character of Cooper 
pairing and a Pomeranchuk instability in the $\ell=1$ spin channel. Our model is based on a 2D Fermi liquid with interactions in the $F_{1}^{a}$ channel and a general attracting interaction favoring Cooper pairing. The main results are summarized in the phase diagram of figure (1), obtained in a self-consistent mean-field approximation described below. For $F_{1}^{a}>-2$, the system is in the usual s-wave SC state. When $F_{1}^{a}<-2$ the Pomeranchuk instability sets in and we observe two types of superconducting phases. At low coupling $g$, there is a splitting of the Fermi surface into two branches, labeled by helicity eigen-values and, the mean field solution is a p-wave superconducting state coming from intra-band pairing. Notice that the pairing is in the helicity basis, since the spin in no longer a good quantum number. The use of the term p-wave refers to the symmetry of the superconducting gap obtained. For greater coupling, Pomeranchuk instability is suppressed continuously and the s-wave superconducting state reappears discontinuously at the bold line. Therefore, at mean field level (and zero temperature) there is a first order phase transition between two different SC states. Moreover, for a stronger BCS coupling, a modulated superconducting phase could appear with a finite wave-vector $\vec{q}$, given roughly by $|\vec{q}| \sim k_{F} \sqrt{1-2 /\left|F_{1}^{a}\right|}$, produced by inter-band BCS couplings. However, this phase is metastable at mean field level.

In the rest of the paper we present our model and sketch the mean-field calculation, leading to the phase diagram of fig. (11).

\section{Model Hamiltonian}

The $\beta$-phase order parameter is given by

$$
V^{a \mu}(x)=-i \psi^{\dagger}(x) \sigma^{\mu} \hat{\nabla}_{a} \psi(x),
$$

where $\psi$ is a two-component spinor, $\sigma^{\mu}$ are the Pauli matrices, and $a=x, y . \hat{\nabla} e^{i k x}=$ $i \frac{\vec{k}}{|k|} e^{i k x}$.

Following ref. [12], we write a Hamiltonian with forward two-body interactions in this channel

$$
\begin{aligned}
H & =\int d^{2} x \psi^{\dagger}(x)(\epsilon(-i \nabla)-\mu-h \vec{\sigma} \cdot \vec{\nabla}) \psi(x)+ \\
& +\frac{1}{2} \int d^{2} x d^{2} x^{\prime} f_{1}^{a}\left(x-x^{\prime}\right) V^{a \mu}(x) V^{a \mu}\left(x^{\prime}\right) .
\end{aligned}
$$

The Fourier transform of $f_{1}^{a}(x)$ is given by $f_{1}^{a}(k)=f_{1}^{a} /\left(1+\kappa\left|f_{1}^{a}\right| k^{2}\right)$, defining, in this way, an effective interaction range $r=\sqrt{\kappa\left|f_{1}^{a}\right|}$. We have considered, in the quadratic part of the Hamiltonian, an explicit spin-orbit (SO) coupling with strength $h$. This term will serve to compute the SO susceptibility and we may eventually take $h \rightarrow 0$. It will also be useful to control the stability of the Pomeranchuk $\beta$-phase. For a particle-hole symmetric system we consider the following expansion of the dispersion relation around a circular Fermi surface,

$$
\epsilon(k)-\mu=\vec{v}_{F} \cdot\left[\vec{k}-\vec{k}_{F}\right]+\frac{b}{\left(v_{F} k_{F}\right)^{2}}\left(\vec{v}_{F} \cdot\left[\vec{k}-\vec{k}_{F}\right]\right)^{3},
$$




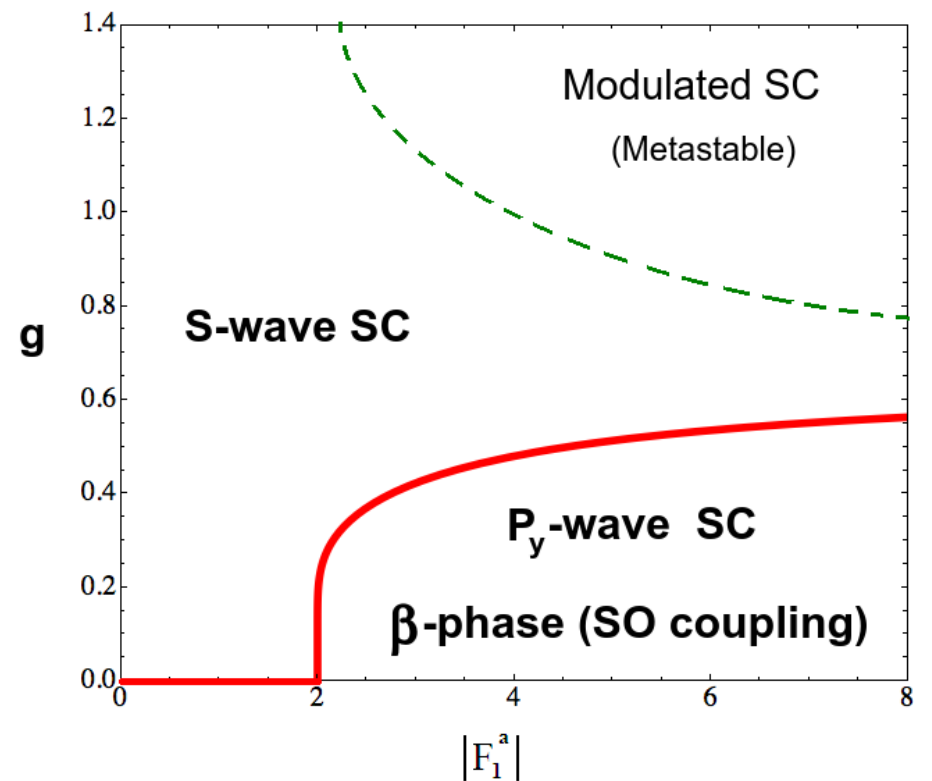

Fig. 1. Phase diagram in terms of the BCS coupling constant $g$ and the dimensionless antisymmetric Landau parameter with angular momentum $\ell=1, F_{1}^{a}=f_{1}^{a} N(0)$. Above the solid line, the system is mainly in an s-wave superconducting state $\left(\bar{n}=0, \Delta_{0} \neq 0, \Delta=0\right)$. Below this line, for $F_{1}^{a}<-2$ and for small $g$, the system continuously develops an spontaneous spin-orbit interaction, given by the order parameter $\bar{n}$. The s-wave order parameter goes to zero discontinuously at this line, where a superconducting order with $p_{y}$-wave symmetry, coexisting with a spin-orbit coupling ( $\beta$-phase) appears. In this phase $\bar{n} \neq 0, \Delta_{0}=0, \Delta \neq 0$. For stronger couplings, the dashed line indicates the onset of a metastable modulated SC phase $\left(\Delta_{q} \neq 0\right)$.

where the dimensionless parameter $b$ measures the effective curvature of the band near the Fermi surface and we have ignored terms proportional to $\left(k-k_{F}\right)^{5}$. The band curvature in the dispersion relation is essential to stabilize any Pomeranchuk instability ${ }^{5}$, for this reason, the usual linear approximation is not suitable to study this type of phase transitions.

The $\beta$-phase is defined by the mean-field Hamiltonian

$$
H_{\beta}=\int \frac{d^{2} k}{(2 \pi)^{2}} \psi^{\dagger}(k)(\epsilon(k)-\mu-(\bar{n}+h) \vec{\sigma} \cdot \hat{k}) \psi(k),
$$

where $\bar{n}$ is determined self-consistently by

$$
\bar{n}=-\frac{1}{2} f_{1}^{a}(0) \int \frac{d^{2} k}{(2 \pi)^{2}}\left\langle\psi^{\dagger}(k)(\vec{\sigma} \cdot \hat{k}) \psi(k)\right\rangle .
$$

This mean-field theory is valid when $k_{F} \sqrt{\kappa\left|f_{1}^{a}\right|} \gg 1, i$. e., when the range of the interaction is much larger than the inter-particle distance.

It is not difficult to solve eq. (5), by considering a Fermi liquid ground state 12,13 . When $\lim _{h \rightarrow 0} \bar{n}(h) \neq 0$, the spectrum of $H_{\beta}$ spontaneously splits into two opposite 


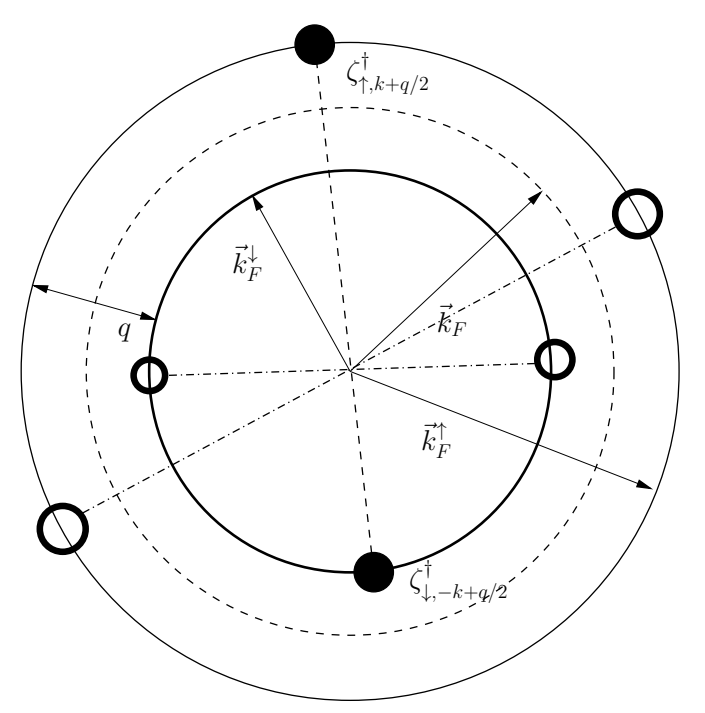

Fig. 2. Fermi surface splitting in the helicity basis and pairing in the $\beta$-phase. The full dots indicate inter-band Cooper pairing with momentum $q$, while the empty dots represent intra-band p-wave Cooper pairing.

chiralities with dispersions $\epsilon^{\uparrow}=\epsilon(k)-(\mu+\bar{n})$ and $\epsilon^{\downarrow}=\epsilon(k)-(\mu-\bar{n})$, as shown in figure (2). The Fermi momentum of each branch is given by $k_{F}^{\uparrow \downarrow}=k_{f} \pm q / 2$ and the relation between $q$ and $\bar{n}$ is computed using eq. (3),

$$
\frac{v_{F} q}{2}=\bar{n}-\frac{b}{\left(v_{F} k_{F}\right)^{2}} \bar{n}^{3}+O\left(\bar{n}^{5}\right)
$$

which is valid close to the Pomeranchuk instability. To obtain this relation we have fixed the chemical potential. A different result is obtained by considering, instead, a fixed density $\underline{\underline{13}}$.

The ordered phase is characterized by a spontaneously generated spin-orbit coupling with the global rotation invariance unbroken. The spin and orbital angular momentum are not conserved independently, however the total angular momentum $\vec{J}=\vec{L}+\vec{S}$ is conserved. In the helicity basis $\zeta=\left(\zeta_{\uparrow}, \zeta_{\downarrow}\right)$, where the operator $\hat{k} \cdot \vec{\sigma}$ is diagonalized with eigenvalues \pm 1 , the mean-field Hamiltonian takes the simpler form

$$
H_{\beta}=\int \frac{d^{2} k}{(2 \pi)^{2}} \zeta^{\dagger}(k)\left(\epsilon(k)-\mu-(\bar{n}+h) \sigma^{z}\right) \zeta(k),
$$

with the self-consistent equation

$$
\bar{n}=-\frac{1}{2} f_{1}^{a}(0) \int \frac{d^{2} k}{(2 \pi)^{2}}\left\langle\zeta^{\dagger} \sigma^{z} \zeta\right\rangle .
$$

Therefore, if $\bar{n} \neq 0$ (with $h=0$ ), there is a dynamically generated spin-orbit coupling. The mean field Hamiltonian studied in this example is similar to the 2D 
Rashba spin-orbit coupling, characterized by a ground state in which the spin configuration has a vortex structure in momentum space 13 with winding number $w=1$. In the Pomeranchuk transition it is also possible to obtain a Dresselhaus spin-orbit coupling in which the vortex structure has winding number $w=-1$. However, both couplings produce the same splitting of the Fermi surface as described in fig. (2). Therefore, the study of the superconducting instability is completely analog and the phase diagram is the same of figure (10) in both cases.

A BCS type interaction in the $\psi, \psi^{\dagger}$ basis induces different types of interactions in the $\beta$-phase, where the usual Fermi liquid properties are lost. In the helicity basis $\zeta, \zeta^{\dagger}$, intra-band p-wave pairing, as well as inter-band finite momentum pairing are induced, as shown in figure (2). These interactions are competing with each other and with interactions in the forward scattering (Pomeranchuk) channel.

The inter-band pairing can be modeled by considering the following attractive interaction favoring Cooper-pair formation with linear momentum $\vec{q}$,

$$
H_{\text {Inter }}=\int \frac{d^{2} k}{(2 \pi)^{2}} \Delta_{q} \zeta_{\uparrow, k+q / 2}^{\dagger} \zeta_{\downarrow,-k+q / 2}^{\dagger}+\text { h.c. },
$$

Note that $\vec{q}$ (eq. (6) ) is given by the Pomeranchuk instability and should be determined self-consistently. The "gap equation" that complements eq. (9) is

$$
\Delta_{q}=-g \int \frac{d^{2} k}{(2 \pi)^{2}}\left\langle\zeta_{\downarrow,-k+q / 2} \zeta_{\uparrow, k+q / 2}\right\rangle
$$

On the other hand, intra-band pairing can be studied by considering

$$
H_{\text {Intra }}=\int \frac{d^{2} k}{(2 \pi)^{2}}\left\{\Delta_{\uparrow}(k) \zeta_{\uparrow, k}^{\dagger} \zeta_{\uparrow,-k}^{\dagger}+\Delta_{\downarrow}(k) \zeta_{\downarrow, k}^{\dagger} \zeta_{\downarrow,-k}^{\dagger}\right\}+\text { h.c. }
$$

where the simplest representation for the gap function is $\Delta_{\uparrow \downarrow}(k)=\Delta_{\uparrow \downarrow} \sin (\theta)$ with $\left(k_{x}, k_{y}\right)=k(\cos \theta, \sin \theta)$, in such a way that $\Delta_{\uparrow \downarrow}(k)=-\Delta_{\uparrow \downarrow}(-k)$. The self consistent constraint that complements this mean field Hamiltonian is

$$
\Delta_{\uparrow \downarrow}=-g \int \frac{d^{2} k}{(2 \pi)^{2}} \sin \theta\left\langle\zeta_{\uparrow \downarrow, k} \zeta_{\uparrow \downarrow,-k}\right\rangle .
$$

Thus, $\Delta_{\uparrow \downarrow}$ is the order parameter of a $p_{y}$-wave SC phase. The $p_{x}$ component, proportional to $\cos \theta$, exactly cancels due to the anti-commuting character of the fermionic operators in Eq. (11). This is due to the simple symmetry of the form factors used to define the BCS couplings.

\section{Self-consistent solution}

To find the phase diagram of figure (11) we need to solve the self-consistent equations (8), (10) and (12) where the expectation values should be computed with the meanfield Hamiltonian $H=H_{\beta}+H_{\text {Intra }}+H_{\text {Inter given by equations (7), (9) and (11). }}$. 
We used the Green's function method to compute expectation values,

$$
\langle B A\rangle=\int d \omega f(\omega) \operatorname{Im}(\ll B ; A \gg)
$$

where $A$ and $B$ are two fermionic operators and $f(\omega)$ is the Fermi function. The notation $\ll A ; B \gg$ indicates the Green's function associated with these operators and can be computed using the equation of motion for Green's functions which, in frequency representation, reads 26 ,

$$
\omega \ll A ; B \gg=\ll[A, H] ; B \gg+\langle\{A, B\}\rangle
$$

Since $H$ is quadratic, equation (14) has closed solutions. We can compute for instance,

$$
\ll \zeta_{\uparrow, k} ; \zeta_{\uparrow, k}^{\dagger} \gg=\frac{\left(\omega+\epsilon_{k}^{\uparrow}\right)\left(\omega+\epsilon_{k-q}^{\downarrow}\right)}{\left(\omega+\epsilon_{k-q}^{\downarrow}\right)\left[\omega^{2}-\left(\epsilon_{k}^{\uparrow}\right)^{2}-\Delta^{2}(\theta)\right]-\Delta_{q}^{2}\left(\omega+\epsilon_{k}^{\uparrow}\right)},
$$

where, for simplicity, we assumed $\Delta_{\uparrow}=\Delta_{\downarrow}=\Delta$. Of course, this procedure is completely equivalent to the diagonalization of the Hamiltonian by means of a Bogoliuvov transformation.

Single particle excitations are given by the poles of the Green's function. In our case this is a cubic polynomial and can be easily computed. For weak couplings, $i$. e. , for infinitesimal BCS coupling, we cannot have modulated superconductivity $\underline{\underline{19}}$. Therefore, in the region where the Pomeranchuk instability sets in, and for small values of the coupling constant, the only possible solution of equation (10) is $\Delta_{q}=0$. Therefore, in this regime, both helicity branches are decoupled, and single particle excitations have the dispersion relation

$$
\omega^{2}=\left(\epsilon_{k}^{\uparrow \downarrow}\right)^{2}+\Delta^{2}(\theta)
$$

Taking the imaginary part of the Green's function and using equation (13) to compute expectation values, we can rewrite equation (8) as

$$
\begin{aligned}
\bar{n} & =-\frac{1}{2} f_{1}^{a}(0) \int \frac{d^{2} k}{(2 \pi)^{2}}\left(\left\langle\zeta_{\uparrow}^{\dagger} \zeta_{\uparrow}\right\rangle-\left\langle\zeta_{\downarrow}^{\dagger} \zeta_{\downarrow}\right\rangle\right) \\
& =-\frac{1}{2} f_{1}^{a}(0) \int \frac{d^{2} k}{(2 \pi)^{2}}\left(\frac{\epsilon_{k}^{\uparrow}}{\sqrt{\left(\epsilon_{k}^{\uparrow}\right)^{2}+\Delta^{2}(\theta)}}-\frac{\epsilon_{k}^{\downarrow}}{\sqrt{\left(\epsilon_{k}^{\downarrow}\right)^{2}+\Delta^{2}(\theta)}}\right)
\end{aligned}
$$

Performing the integrals, and considering negative values of $f_{1}^{a}(0)$ we find,

$$
\bar{n}=\frac{1}{2}\left|F_{1}^{a}\right|\left(1+8\left(\frac{\pi \Delta}{2 \Lambda}\right)^{2} \ln \left(\frac{\pi \Delta}{2 \Lambda}\right)\right) \bar{n}\left[1-\frac{b}{\left(v_{F} k_{F}\right)^{2}} \bar{n}^{2}\right]
$$

with $F_{1}^{a}=f_{1}^{a} N(0)$, where $N(0)$ is the density of states at the Fermi surface, and $\Lambda$ is an ultraviolet momentum cut-off. In the absence of pairing $\Delta=0$, this equation 
has non trivial solutions $\bar{n} \neq 0$ for $F_{1}^{a}<-2$, signaling the usual Pomeranchuk instability. The presence of pairing restricts this region to

$$
\left|F_{1}^{a}\right|>\frac{2}{1+8\left(\frac{\pi \Delta}{2 \Lambda}\right)^{2} \ln \left(\frac{\pi \Delta}{2 \Lambda}\right)}
$$

On the other hand, within this region, the two helicity branches are decoupled and the gap equation for $p_{y}$-wave pairing, equation (12) reads,

$$
\Delta=-g \int \frac{d^{2} k}{(2 \pi)^{2}} \frac{\Delta \sin ^{2} \theta}{\sqrt{\epsilon_{k}^{2}+\Delta^{2} \sin ^{2} \theta}}
$$

which, due to the usual logarithmic divergence, has a non-trivial solution $\Delta \sim$ $\Lambda e^{-1 / g}$. Therefore, at mean-field level, the system develops a p-wave superconductivity coexisting with a spin-orbit interaction in a small region of the phase diagram under the bold line in figure (1). As can be seen from equation (18), the line

$$
\left|F_{1}^{a}\right|=\frac{2}{1+2 \pi^{2} e^{-2 / g} \ln \left[(\pi / 2) e^{-1 / g}\right]}
$$

represents a continuous Pomeranchuk transition between $\bar{n} \neq 0$ and $\bar{n}=0$. Above this transition, there is no more splitting of the Fermi surface, since $q=0$, and the character of the ground state changes qualitatively.

Above the Pomeranchuk line, Eq. (21), the one-particle excitations are given now by,

$$
\omega^{2}=\epsilon_{k}^{2}+\Delta_{0}^{2}+\Delta^{2} \sin ^{2} \theta
$$

as can be seen from the structure of the Green's function, Eq. (15), by taking $q \rightarrow 0$. In this regime, the gap equations (10) and (12) are coupled,

$$
\begin{aligned}
\Delta & =-g \int \frac{d^{2} k}{(2 \pi)^{2}} \frac{\Delta \sin ^{2} \theta}{\sqrt{\epsilon_{k}^{2}+\Delta_{0}^{2}+\Delta^{2} \sin ^{2} \theta}} \\
\Delta_{0} & =-g \int \frac{d^{2} k}{(2 \pi)^{2}} \frac{\Delta_{0}}{\sqrt{\epsilon_{k}^{2}+\Delta_{0}^{2}+\Delta^{2} \sin ^{2} \theta}} .
\end{aligned}
$$

The only solution of these equations above the curve $g\left(F_{1}^{a}\right)$ (Eq. (21)) is $\Delta=0$, $\Delta_{0} \sim e^{-1 / g}$. For this reason, the bold curve in the phase diagram represents a first order transition between two superconducting states: the "normal" s-wave superconductor and a $p_{y}$-wave superconductor state characterized by a spin-orbit coupling responsible for the helicity splitting of the Fermi surface. Note that, despite that pairing is taking place in the helicity basis, we use the terms s-wave and p-wave to denote different symmetries of order parameters. We stress that, the $p_{y}$-wave order parameter in the $\beta$-phase, when written in terms of the original Cartesian basis, is a mixture with other partial wave channels, including s-wave.

The only interesting possibility we have left aside is the presence of modulated superconductivity due to the spontaneous Fermi surface splitting, produced by a Pomeranchuk instability. In the next subsection we analyze this possibility. 


\subsection{Looking for modulated superconductivity}

We would like to look for a solution of the self consistent equations of the form $\bar{n} \neq 0, \Delta_{q} \neq 0$ and $\Delta=0$. In this case, the single particle excitations are,

$$
\omega_{ \pm}=\left(\frac{\epsilon_{k+q / 2}^{\uparrow}-\epsilon_{k-q / 2}^{\downarrow}}{2}\right) \pm \xi,
$$

with

$$
\xi=\sqrt{\left(\frac{\epsilon_{k+q / 2}^{\uparrow}+\epsilon_{k-q / 2}^{\downarrow}}{2}\right)^{2}+\left|\Delta_{q}\right|^{2}} .
$$

The gap equations (8) and (10) are written now as (at zero temperature)

$$
\begin{gathered}
\bar{n}=\frac{\left|f_{1}^{a}\right|}{4} \int \frac{d^{2} k}{(2 \pi)^{2}}\left\{\Theta\left(-\omega_{+}\right)-\Theta\left(\omega_{-}\right)\right\}, \\
\Delta_{q}=-g \int \frac{d^{2} k}{(2 \pi)^{2}} \frac{\Delta_{q}}{2 \xi}\left\{\Theta\left(-\omega_{+}\right)-\Theta\left(-\omega_{-}\right)\right\},
\end{gathered}
$$

where $\Theta$ is the usual Heaviside function. It is simple to realize that the s-wave BCS order parameter $\bar{n}=0, \Delta_{q}=\Delta_{0}$ is always a solution of eqs. (27) and (28).

Now, we explore the interesting possibility of a modulated solution $\bar{n} \sim q \neq$ $0, \Delta_{q} \neq 0$. The $\vec{k}$-integrals in equations (27) and (28) are strongly constrained by the Heaviside functions. The main contribution to eq. (27) comes from the region $\omega_{+}<0$. Written in polar coordinates $(k, \theta)$, with $\cos \theta=(\vec{k} \cdot \vec{q}) / k q$, and first integrating over $k$, we get

$$
\begin{aligned}
\bar{n} & =\left|F_{1}^{a}\right| \bar{n} \int_{\theta^{-}}^{\theta^{+}} \frac{d \theta}{2 \pi} \times \\
& \times \sqrt{\left(1-\frac{b}{\left(v_{F} k_{F}\right)^{2}} \bar{n}^{2} \cos \theta\right)^{2}(1+\cos \theta)^{2}-x^{2}}
\end{aligned}
$$

where we have defined the dimensionless quantity $x=\Delta_{q} / \bar{n} \cdot \theta^{ \pm}(x, \bar{n})$ are the integration limits which keep the argument of the square root positive. $\left|F_{1}^{a}\right|=$ $N(0) f_{1}^{a}$, where $N(0)$ is the density of states at the original Fermi surface (with $\bar{n}=0$ ). This approximation is valid very near the critical point where the number of states at the split Fermi surfaces are essentially equal. Near the critical point, we can expand the integrand in powers of $\bar{n} / v_{F} k_{F}<<1$, obtaining

$$
\bar{n}=\frac{\left|F_{1}^{a}\right|}{2} \bar{n}\left[C_{0}(x)-\frac{b}{\left(v_{F} k_{F}\right)^{2}} \bar{n}^{2} C_{1}(x)\right]+O\left(\left(\frac{\bar{n}}{v_{F} k_{F}}\right)^{5}\right),
$$

with $C_{0}(x) \sim 1-(x / 2)^{3 / 2}, C_{1}(x)=1+x \ln (1+x / 5)$ and $F_{1}^{a}=f_{1}^{a} N(0)$, where $N(0)$ is the density of states at the Fermi surface. 
On the other hand, the integral in eq. (28) has the usual ultraviolet divergence of the BCS gap equation. A convenient way to deal with this integral is to sum and subtract $\Theta\left(\omega_{-}\right)$in the second term and then to subtract the identity

$$
-1+g \int \frac{d^{2} k}{(2 \pi)^{2}} \frac{1}{2 \xi^{0}} \equiv 0
$$

from the first term of eq. (28). $\xi^{0}=\sqrt{\epsilon_{k}^{2}+\left|\Delta_{0}\right|^{2}}$ and $\Delta_{0}$ is the uniform superconducting gap in the absence of Fermi surface splitting. With this, eq. (28) is rewritten as

$$
\frac{N(0)}{2} \ln \left|\frac{\Delta_{0}}{\Delta_{q}}\right|=\int \frac{d^{2} k}{(2 \pi)^{2}} \frac{1}{2 \xi}\left\{\Theta\left(-\omega_{+}\right)+\Theta\left(\omega_{-}\right)\right\} .
$$

In this way, the above integral is controlled. The coupling constant $g$ and the ultraviolet cut-off are both contained in the definition of $\Delta_{0}$ (eq. (31)). As before, near the Fermi surface, where interactions are important, $\Theta\left(\omega_{-}\right)=0$. Performing the remaining integral over $k$, the gap equation is written as

$$
\ln \left|\frac{\Delta_{0}}{\Delta_{q}}\right|=\Gamma(x),
$$

where $x=\Delta_{q} / \bar{n}$, and we have defined the function

$$
\Gamma(x)=2 \int_{-\bar{\theta}(x)}^{\bar{\theta}(x)} \frac{d \theta}{2 \pi} \sinh ^{-1}\left(\frac{\sqrt{(1+\cos \theta)^{2}-x^{2}}}{x^{2}}\right),
$$

with $\bar{\theta}(x)=\cos ^{-1}(x-1)$ for $x \leq 2$ and $\Gamma(x>2)=0$. Therefore, to look for modulated superconductivity we need to solve eqs. (30) and (33) self-consistently. Firstly, we note that for $x>2$ the only solution, $\left\{\bar{n}=0, \Delta_{q}=\Delta_{0}\right\}$, corresponds to the uniform superconducting phase. This imposes a lower limit for modulated superconductivity since, for obtaining a non-trivial solution, we must have $\Delta_{q}<$ $2 \bar{n}<\Delta_{0}$. This is consistent with the fact that modulated superconductivity is a strong coupling effect. However, for any value of the parameter $\Delta_{0}>2 \bar{n}$, the uniform superconducting mean-field energy is always lower than the modulated one, $\langle H\rangle_{\Delta_{0}}\left\langle\langle H\rangle_{\Delta_{q}}\right.$. On the other hand, there is a region of the phase diagram, shown above the dashed line in figure (10), in which $\langle H\rangle_{\Delta_{q}}\left\langle\langle H\rangle_{\beta}\right.$. Therefore, modulated superconductivity appears as a metastable phase for large values of $g$ and $\left|F_{1}^{a}\right|>2$. The precise location of the onset of metastability depends on the relation between the band-width in which interactions are relevant (the energy cutoff), and the band-curvature at the Fermi surface. We would like to note that, the meta-stable character of the modulated SC phase may depend on the symmetry of the form factor used to define the effective BCS coupling.

\section{Discussion and Conclusions}

Summarizing, we have studied, in the context of Fermi liquids, the competition between a Pomeranchuk instability in the spin channel with angular momentum 
$\ell=1$, triggered by the Landau parameter $F_{1}^{a}$ and an attractive interaction favoring Cooper-pair formation. We built up a zero temperature mean-field phase diagram in terms of the two parameters of the model: $F_{1}^{a}$ and $g$. We found that, as observed in other channels of Pomeranchuk instabilities ${ }^{24,25}$, the superconducting gap reduces the tendency to Fermi surface deformation. We have considered intra-band homogeneous pairing as well as inter-band pairing. The net effect of the former is to produce a $p_{y}$-wave superconductor state for very small values of the BCS coupling constant. Then, we have shown that, for stronger couplings, a first order p-wave/s-wave phase transition takes place. Moreover, we have found a metastable modulated superconductor, enhanced for larger values of $\left|F_{1}^{a}\right|$.

The phase diagram of figure (1) could be modified by long wave-length fluctuations in several ways. In the $\beta$-phase, there are three branches of Goldstone modes transforming with $S O(3)$ group, associated with the breaking of relative spin-orbit symmetry. The longitudinal mode has a linear dispersion relation, while the transverse modes signal a tendency to produce a non-homogeneous ground state with parity symmetry breaking 13 , meaning that, in the absence of SC interactions, the mean field solution of the $\beta$-phase is actually meta-stable. However, an explicit SO coupling $(h)$ can gapped the Goldstone modes, stabilizing the phase and rounding the Pomeranchuk instability. On the other hand, it is known that a modulated superconductor order parameter can coexist, at strong coupling, with non-homogeneous charge configurations $\frac{19}{}$. This coexistence has also been proposed in the context of cuprates superconductors ${ }^{20}$. For this reason, we believe that transverse long-wave fluctuations of the $\beta$-phase could probably enhance the modulated $\mathrm{SC}$ order.

To the best of our knowledge, there is still no experimental evidence of the phases described in this paper. However, we believe that they could be possibly observed in systems with attractive interactions in the antisymmetric $\ell=1$ channel. Negative values of the Landau parameter $F_{1}^{a}$ have been measure $27,28,29,30$ in systems like ${ }^{3} \mathrm{He}$. Also, in ultra cold atomic gases with a p-wave Feshbach resonance ${ }^{31}, F_{1}^{a}$ should be negative near the resonance. More generally, strongly correlated systems like cuprates, heavy Fermions and two dimensional electron systems in high magnetic fields are an interesting arena to investigate the phenomenology presented in this paper.

\section{Acknowledgments}

D.G.B. is in debt with Eduardo Fradkin for very useful discussions. The Brazilian agencies "Conselho Nacional de Desenvolvimento Científico e Tecnológico", (CNPq) and the "Fundação de Amparo à Pesquisa do Estado do Rio de Janeiro", (FAPERJ), are acknowledged for partial financial support.

\section{References}

1. P. Nozieres and D. Pines, The Theory of Quantum Liquids (Perseus Books, 1999). 
2. I. I. Pomeranchuk, Sov. Phys. JETP 8, (1958) 361.

3. H. Yamase and H. Kohno, J. Phys. Soc. Jpn. 69, (2000) 332; J. Phys. Soc. Jpn. 69, (2000) 2151.

4. V. Oganesyan, S. A. Kivelson, and E. Fradkin, Phys. Rev. B64,(2001) 195109 .

5. D. G. Barci and L. E. Oxman, Phys. Rev. B67, (2003) 205108.

6. M. J. Lawler, D. G. Barci, V. Fernández, E. Fradkin, and L. Oxman, Phys. Rev. B73, (2006) 085101.

7. M. A. Metlitski and S. Sachdev, Phys. Rev. B82, (2010) 075127.

8. D. G. Barci, M. Trobo, V. Fernández, and L. E. Oxman, Phys. Rev. B78, (2008) 035114.

9. Y. Zhang and S. Das Sarma, Phys. Rev. B72, (2005) 115317.

10. J. E. Hirsch, Phys. Rev. B41, (1990) 6820.

11. P. Rodriguez Ponte, D. C. Cabra, N. Grandi, Mod. Phys. Lett. B26, (2012) 12501.

12. C. Wu and S.-C. Zhang, Phys. Rev. Lett. 93, (2004) 036403.

13. C. J. Wu, K. Sun, E. Fradkin, and S.-C. Zhang, Phys. Rev. B75, (2007) 115103.

14. P. A. Frigeri, D.F. Agterberg, M. Sigrist, New J. Phys.6, (2004) 115.

15. P. Fulde and R. A. Ferrell,Phys. Rev. 135, (1964) A550.

16. A. I. Larkin and Y. N. Ovchinnikov, Zh. Eksp. Teor. Fiz. 47,(1964) 1136 , (Sov. Phys. JETP. 20,(1965) 762).

17. L. Radzihovsky and A. Vishwanath, Phys. Rev. Lett. 103, (2009) 010404.

18. I. T. Padilha and M. A. Continentino, Journal of Physics: Condensed Matter 21,(2009) 095603.

19. F. Loder, A. P. Kampf, and T. Kopp, Phys. Rev. B81, (2010) 020511.

20. E. Berg, E. Fradkin, E.-A. Kim, S. A. Kivelson, V. Oganesyan, J. M. Tranquada, and S. C. Zhang, Phys. Rev. Lett.99, (2007) 127003.

21. E. Berg, E. Fradkin, and S. A. Kivelson, Nature Physics 5, (2009) 830.

22. E. Berg, E. Fradkin, S. A. Kivelson, and J. M. Tranquada, New J. Phys. 11,(2009) 115004.

23. D. G. Barci and E. Fradkin, Phys. Rev. B83, (2011) 100509.

24. C. J. Halboth and W. Metzner, Phys. Rev. Lett. 85, (2000) 5162.

25. H. Yamase and W. Metzner, Phys. Rev. B75, (2007) 155117.

26. D. N. Zubarev, Sov. Phys. USPEKHI 3, (1960) 320.

27. A. J. Leggett, J. Phys. C 3, (1970) 448.

28. L. R. Corruccini, D. D. Osheroff, D. M. Lee, and R. C. Richards, Phys. Rev. Lett. 27, (1971) 650.

29. D. D. Osheroff, Physica B \& C 90, (1977) 20.

30. D. S. Greywall, Phys. Rev. B 27, (1883) 2747.

31. J. Zhang, E. G. M. van Kempen, T. Bourdel, L. Khaykovich, J. Cubizolles, F. Chevy, M. Teichmann, L. Tarruell, S. J. J. M. F. Kokkelmans, and C. Salomon, Phys. Rev. A 70, (2004) 030702. 